J. Clin. Chem. Clin. Biochem.

Vol. 14, 1976, pp. 449-452

\title{
Zur Aktivitätsbestimmung von Peptidasen und Aminosäurearylamidasen
}

\author{
Von Th. Unger und $H$. Struck
}

Aus der Biochemischen und experimentellen Abteilung (Leiter: Prof. Dr. H. Struck) am II. Chirurgischen Lehrstuhl (Direktor: Prof. Dr. W. Schink) der Universität zu Köln in Köln-Merheim und dem Ernst-Rodenwaldt-Institut (Leiter: OTA Dr. K. Wiemann) Koblenz

(Eingegangen am 20. März/28. Juni 1976)

Zusammenfassung: In der vorliegenden Arbeit wird eine Modifikation der Bratton-Marshall-Reaktion zum quantitativen Nachweis von enzymatisch freigesetztem $p$-Nitroanilin dargestellt.

Sie ist sehr empfindlich, leicht ausführbar, gut reproduzierbar und als Mikromethode für die Anwendung in der klinisch-chemischen Routine geeignet.

\section{Determination of the activity of peptidases and amino acid aryl amidases}

Summary: A modification of the Bratton-Marshall reaction for the quantitative determination of enzymically released $p$-nitroaniline is described. It is very sensitive, easily performed, shows good reproducibility, and it is suitable for routine use in clinical chemistry.

\section{Einführung}

Im Humanserum befinden sich Peptidasen und Arylamidasen, deren Herkunft, physiologische Funktion und pathognomonische Bedeutung bis auf einige Ausnahmen weitgehend ungeklärt sind.

In neuerer Zeit wurden diese Enzyme jedoch häufiger in gesunden und pathologisch veränderten menschlichen Geweben und Seren bestimmt (1-13); es erscheint daher folgerichtig, sie auf ihre Eignung als diagnostische Parameter zu untersuchen und selbst geringe Aktivitätsund Spezifitätătsunterșchiede im Serum mit Hilfe einer empfindlichen Testmethodik und geeigneten Substraten - hier mit Aminosäure-p-nitroaniliden - nachzuweisen.

Die übliche direkte photometrische Messung des enzymatisch freigesetzten $p$-Nitroanilins bei $405 \mathrm{~nm}$ ist jedoch wegen der geringen Enzymaktivitäten meist nicht möglich; darüber hinaus wird die NitroanilinFarbe von der Eigenfarbe des Serums überlagert.

1939 veröffentlichten Bratton, Marshall und Mitarbeiter (14) eine Methode zum quantitativen Șulfonamidnachweis im Blut, die auf der Diazotierung der freien Aminogruppe des Sulfanilamids. in saurer Lösung und anschließender Kupplung an N-1-Naphthyl-ethylendiamin zu einem violetten Azo-Farbstoff beruht; sie ist prinzipiell auch auf andere Aminobenzol-Derivate und -Homologe und damit auch auf $p$-Nitroanilin anwendbar (15).

In der vorliegenden Arbeit wird nun eine Modifikation der Bratton-Marshall-Reaktion zum quantitativen Nachweis enzymatisch freigesetzten $p$-Nitroanilins mit $\mathrm{N}$-(Napthyl-1(1))-ethylendiammoniumdichlorid als Chromogen dargestellt. Sie ist einfacher, weniger störanfällig und - bezogen auf die $p$-Nitroanilin-Konzentration im Testgemisch - je nach Ausführung etwa dreifach bis fünfzehnfach empfindlicher als die ursprüngliche, bei Appel (16) näher beschriebene Methode; als Mikromethode ist sie auf die Erfordernisse eines Routinelabors abgestimmt.

\section{Material und Methoden .}

Alle Chemikalien wurden von der Firma Merck, Darmstadt, bezogen.

Für die Enzyymteste wurde Poolserum von gesunden weiblichen und männlichen Probanden im Alter von 25 bis $33 \mathrm{Jahren}$ verweñdet, das bei $-20^{\circ} \mathrm{C}$ gelagert und bei Bedarf portionsweise aufgetaut wurde. 
Lösungen

1. p-Nitroanilin-Standard, $0,333 \mathrm{mmol} / \mathrm{l}$ :

13,8 mg 4-Nitroanilin (Merck 6760) werden in $1 \mathrm{ml}$ Dimethylsulfoxid (Merck 2912) gelöst und mit bidest. Wasser auf $300 \mathrm{ml}$ aufgefullt. Die Lösung ist bei $4^{\circ} \mathrm{C}$ unbegrenzt haltbar.

2. Tris-Puffer, $0,1 \mathrm{~mol} / 1 ; \mathrm{pH} 7,6$.

3. Trichloressigsäure, $300 \mathrm{~g} / \mathrm{l}$ :

$30 \mathrm{~g}$ kristalline Trichloressigsäure werden mit bidest. Wasser auf $100 \mathrm{ml}$ aufgefuillt. Bei Raumtemperatur unbegrenzt haltbar.

4. NaCl-Lösung, $0,15 \mathrm{~mol} / 1$.

5. Na-Nitrit, $2 \mathrm{~g} / \mathrm{l}$

$0,2 \mathrm{~g} \mathrm{NaNO}_{2}$ werden in $100 \mathrm{ml}$ bidest. Wasser gelöst. Die Lösung muß bei $4^{\circ} \mathrm{C}$ aufbewahrt und wöchentlich frisch angesetzt werden.

6. Ammoniumamidosulfonat, $5 \mathrm{~g} / \mathrm{l}$ :

$0,5 \mathrm{~g}$ A midosulfonat (Merck 1220) werden in $100 \mathrm{ml}$ bidest. Wasser gelöst. Die Lösung soll ebenfalls im Kühlschrank aufbewahrt und wöchentlich frisch angesetzt werden.

7. N-1-Naphthyl-ethylendiammoniumdichlorid, $0,1 \mathrm{~mol} / 1$ : 0,291 g N-1-Naphthyl-ethylendiammoniumdichlorid (Merck 6237) werden in $10 \mathrm{ml}$ Dimethylsulfoxid gelöst. Die Lösung ist lichtempfindlich und soll täglich frisch bereitet werden.

8. Substrat, $0,1 \mathrm{~mol} / \mathrm{l}$ :

$30,1 \mathrm{mg}$ Tyrosin-p-nitroanilid (Merck 24627) werden in $1 \mathrm{ml}$ Dimethylsulfoxid gelöst. Die Lösung ist bei $4^{\circ} \mathrm{C}$ etwa

30 Tage haltbar. Bei stärkerem Ansteigen der Leerwerte soll sie frisch angesetzt werden.

9. Iso-Amylalkohol p. a. (Merck 979).
Bei größeren Testserien kann die Substratlösung vorteilhaft dem Puffer zugegeben werden, falls das verwendete Aminosäure-p-nitroanilid wasserlöslich ist. Das im Testgemisch enthaltene bidest. Wasser dient zunächst der Verdünnung des Ansatzes, kann aber bei Bedarf durch Lösungen von Effektoren ersetzt werden. Untersuchungen dariber sind im Gange.

\section{Makromethode}

Sind sehr geringe Enzymaktivitäten und damit sehr kleine Mengen freigesetztes $p$-Nitroanilin zu erwarten, so kann man eine Makromethode anwenden, die gegenüber der oben beschriebenen Mikromethode etwa funffach empfindlicher ist. Sie beruht auf der guten Löslichkeit des gebildeten Azo-Farbstoffes in IsoAmylalkohol und gestattet den Nachweis einer $p$-NitroanilinKonzentration im Testgemisch von 0,83 bis $8,3 \mu \mathrm{mol} / \mathrm{l}$. Man geht dazu von den 5 fachen Volumina der Mikromethode aus und überführt den gebildeten Azo-Farbstoff durch Schütteln quantitativ in $1 \mathrm{ml}$ Iso-Amylalkohol. Nach 10minütigem Stehen wird der Ansatz $5 \mathrm{~min}$ bei $3500 \mathrm{U} / \mathrm{min}$ zentrifugiert, die Oberphase entnommen und in Halbmikroküvetten von $1 \mathrm{~cm}$ Schichtdicke gemesssen. Diese Methode erfordert jedoch einigen Arbeitsaufwand und ist daher für die Routine kaum geeignet.

\section{Ergebnisse}

\section{Spektrum}

Die maximale Lichtabsorption des Azo-Farbstoffes im Reaktionsgemisch liegt bei einer Wellenlänge von $550 \mathrm{~nm}$.

Mikromethode, Pipettierschema

\begin{tabular}{|c|c|c|c|c|}
\hline In Reaktionsgefäße pipettieren $(\mu \mathrm{l})$ : & Leerwert & Standard & Probe & Konzentration im Testgemisch \\
\hline p-Nitroanilin-Standard & - & $10-100$ & - & $4,1-41 \mu \mathrm{mol} / 1$ \\
\hline Serum & - & - & $10-200$ & \\
\hline $\mathrm{NaCl} 0,15 \mathrm{~mol} / \mathrm{l}$ & - & ad 200 & ad 200 . & \\
\hline bidest. Wasser & 100 & 100 & 100 & \\
\hline Tris-Puffer $0,1 \mathrm{~mol} / 1 \mathrm{pH} \mathrm{7,6}$ & 500 & 500 & 500 & $62,5 \mathrm{mmol} / 1$ \\
\hline Substrat-Lösung $0,1 \mathrm{~mol} / 1$ & .10 & - & 10 & $1,25 \mathrm{mmol} / 1$ \\
\hline \multicolumn{5}{|c|}{ mischen; $30 \mathrm{~min}$ bei $37^{\circ} \mathrm{C}$ auf einem Thermostaten inkubieren } \\
\hline Trichloressigsäure $300 \mathrm{~g} / 1$ & 500 & 500 & 500 & $187,5 \mathrm{~g} / \mathrm{l}$ \\
\hline Serum & $10-200$ & - & - & \\
\hline $\mathrm{NaCl} 0,15 \mathrm{~mol} / 1$ & ad 200 & - & - & \\
\hline \multicolumn{5}{|c|}{ mischen; $5 \mathrm{~min}$ bei Raumtemperatur stehen lassen; $2 \mathrm{~min}$ bei $15000 \mathrm{U} / \mathrm{min}$ zentrifugieren } \\
\hline In neue Reaktionsgefäße pipettieren $(\mu l)$ : & Leerwert & Standard & Probe & Konzentration im Reaktionsgemisch \\
\hline Überstand & 1000 & 1000 & 1000 & \\
\hline Na-Nitrit $2 \mathrm{~g} / \mathrm{l}$ & 100 & 100 & 100 & $0,15 \mathrm{~g} / 1$ \\
\hline \multicolumn{5}{|l|}{3 min auf einem Rotationsmischer mischen } \\
\hline Amidosulfonat $5 \mathrm{~g} / \mathrm{l}$ & 100 & 100 & 100 & $0,38 \mathrm{~g} / 1$ \\
\hline 3 min auf einem Rotationsmischer mischen & & 。 & & \\
\hline Naphthyl-ethylen-diammoniumchlorid $0,1 \mathrm{~mol} / 1$ & 100 & 100 & 100 & $7,7 \mathrm{mmol} / 1$ \\
\hline
\end{tabular}


Das Molekulargewicht des gebildeten Azo-Farbstoffes beträgt 441,34; der mikromolare Absorptionskoeffizient liegt unter den obengenannten Bedingungen bei $\epsilon=53,8$.

\section{Standardkurve}

Tab. 1. Mittelwerte, Standardabweichungen, Variationskoeffizienten der Mikromethode.

$10-100 \mu l p$-Nitroanilin-Standard $0,33 \mathrm{mmol} / \mathrm{l}$

$A_{550}$, keine Zugabe von Substrat.

Jeder Wert ist das arithmetische Mittel aus fünf Einzelmessungen. Meßwerte, Standardabweichungen und Variationskoeffizienten für die Mikromethode sind tabellarisch dargestellt. Die Absorptionen sind den Konzentrationen proportional; das LambertBeer'sche Gesetz ist für Mikro- und Makromethode guiltig.

\begin{tabular}{llll}
\hline$\mu \mathrm{l}$ & $\overline{\mathrm{x}}$ & $\mathrm{s}$ & $\mathrm{VK}[\%]$ \\
\hline 10 & 0,104 & 0,0037 & 3,5 \\
20 & 0,201 & 0,0043 & 2,2 \\
30 & 0,301 & 0,0055 & 1,8 \\
40 & 0,401 & 0,0072 & 1,8 \\
50 & 0,503 & 0,0081 & 1,6 \\
60 & 0,600 & 0,0101 & 1,7 \\
70 & 0,695 & 0,0168 & 2,4 \\
80 & 0,796 & 0,0164 & 2,1 \\
90 & 0,900 & 0,0115 & 1,3 \\
100 & 1,010 & 0,0170 & 1,7 \\
\hline
\end{tabular}

Einfluß der Nitrit- und Sulfamatkonzentration auf Linearität und Farbentwicklung

Die Nitritkonzentration hatte innerhalb des Intervalls von $0,36-0,09 \mathrm{~g} / 1$ Reaktionsgemisch keinen wesentlichen Einfluß auf die Linearität und die Farbentwicklung; die Konzentration von $0,15 \mathrm{~g} / 1$ wurde beibehalten, um sicherzustellen, daß bei eventuell ablaufenden nitritverbrauchenden Nebenreaktionen dieses noch im Überschuß vorhanden war.

Die Sulfamatkonzentration wurde in Analogie zur ursprünglichen Methode (17) so bemessen, daß nach Abschluß der Diazotierung überschüssiges Nitrit schnell und sicher zerstört wurde.

\section{Einfluß der Chromogenkonzentration}

Die Änderung der Chromogenkonzentration im Reaktionsgemisch zwischen 4 und $15 \mathrm{mmol} / 1$ hatte ebenfalls keinen Einfluß auf die Linearität und die Farbintensität des Tests. Die gegenüber der Ażo-NitroanilinKonzentration von maximal $0,03 \mathrm{mmol} / 1$ weit überschüssige Chromogenkonzentration von $7,7 \mathrm{mmol} / 1$ wurde gewählt, damit ein rascher und vollständiger Ablauf der Kopplungsreaktion gewährleistet blieb.

\section{Enzymaktivität im Serum}

Mit der oben beschriebenen Mikromethode und Tyrosinp-nitröanilid als Substrat wurden Enzymbestimmungen in einem Poolserum (s. Material und Methoden) durchgefuihrt. Tyrosin- $p$-nitroanilid dient hier lediglich als Beispiel und kann durch andere Aminosäure-p-nitroanilide ersetzt werden. Wegen der hohen Empfindlichkeit der Methode wurde das Serum mit $0,15 \mathrm{~mol} / \mathrm{l} \mathrm{NaCl} 10 \mathrm{fach}$ vorverdünnt.
Die höchsten Umsatzraten fanden sich in $0,1 \mathrm{~mol} / 1$ Trispuffer bei einer Temperatur von $37^{\circ} \mathrm{C}$ in einem pH-Bereich zwischen 7,6 und 7,9; die Michaelis-Konstante beträgt unter diesen Bedingungen 0,33 mmol/l.

Die Enzymaktivität des Serums gegenüber Tyrosin-pnitroanilid wurde mit 3,3 U/1 errechnet; das entspricht etwa dem von Lorentz (12) angegebenen Wert von $3,8 \mathrm{U} / 1$.

\section{Präzision in der Serie}

Um die Präzision der Mikromethode in der Serie zu ermitteln, wurden 20 Enzymbestimmungen mit 10 fach vorverdünntem Poolserum in $0,1 \mathrm{~mol} / 1$ Trispuffer $\mathrm{pH} 7,6$ bei $37^{\circ} \mathrm{C}$ und 30 minütiger Inkubationsdauer durchgeführt. Der Mittelwert der bei $550 \mathrm{~nm}$ gemessenen Absorptionen betrug 0,600 bei einer Standardabweichung von $\pm 0,015$ und einem Variationskoeffizienten $\mathrm{VK}=2,5 \%$.

\section{Diskussion}

Die hier beschriebene Methodik gestattet durch ihre hohe Empfindlichkeit den Nachweis sehr geringer Enzymaktivitäten gegenüber Aminosäure-p-nitroaniliden im Serum und anderen Körperflüssigkeiten. Bei gleichen $p$-Nitroanilin-Konzentrationen im Testansatz liefert die hier dargestellte Methode durch Vermeidung der Volumenexpansion gegenüber der bei Appel (16) beschriebenen Originalmethode auf das Dreifache erhöhte Extinktionen, darüber hinaus ist sie zur Anwendung in der klinisch-chemischen Routinediagnostik geeignet.

In der vorliegenden Arbeit fand Tyrosin-p-nitroanilid als Substrat nur deshalb Verwendung, weil es unter herkömmlichen Testbedingungen sehr langsam umgesetzt wird; Lorentz und Mitarbeiter (12) beobachteten eine Spaltung von Tyrosin-p-nitroanilid von 3,8 U/l. Enzymaktivitäten dieser Größenordnung liegen bei der herkömmlichen Methode der direkten photometrischen Messung des freigesetzten $p$-Nitroanilins bei $405 \mathrm{~nm}$ im Bereich der unteren Nachweisgrenze und sind daher nur mit einer relativ großen Fehlerbreite zu bestimmen, während bei Anwendung der hier beschriebenen Methodik bereits eine mindestens 10 fache, besser 20 fache Vorverdünnung des Serums erforderlich wird. Die mögliche direkte Messung sehr geringer Mengen enzymatisch freigesetzten $p$-Nitroanilins mit Hilfe von Schreibern mit Skalenspreizung erfordert dagegen einen apparativen Aufwand, der für die meisten Routinelabors zu groß sein dürfte.

Zur Vermeidung enzyminhibierender Wirkungen des Lösungsmittels Dimethylsulfoxid wurde die Menge der Substratlösung möglichst klein gehalten. Dennoch ergibt sich bei einem Inkubationsvolumen von $810 \mu$ leine Substratkonzentration von $1,25 \mathrm{mmol} / \mathrm{l}$ im Testgemisch; das entspricht etwa der von Nagel (18) angegebenen 
Leucin-p-nitroanilid-Konzentration von $0,8 \mathrm{mmol} / \mathrm{l}$ zur Messung der Leucin-Aminopeptidase. Bei einer MichaelisKonstanten von $0,33 \mathrm{mmol} / \mathrm{l}$ für Tyrosin-p-nitroanilid finden die Enzymbestimmungen demnach bei ausreichender Substratkonzentration statt. Bèi der Verwendung anderer Aminosäure-p-nitroanilide dürften ähnliche Verhältnisse vorliegen.

Auf die Spaltung anderer Aminosäure-p-nitroanilide, deren Beeinflussung durch Effektoren und die șich

\section{Literatur}

1. Scherstén, T., Wahlqvist, L. \& Johannson, L. G. (1969), Cancer 23, 608-613.

2. Scherstén, T., Wahlqvist, L. \& Jilderos, B. (1971), Cancer $27,278-283$.

3. Scherstén, T. \& Lundholm, K. (1972), Cancer 30, 1246 -1251 .

4. Gries, G. \& Strauch, C. (1970), Klin. Wochenschr. 48, 441-442.

5. Stein, U., Heismeier, H., Zimmermann, W. \& Lesch, R. (1971), Klin. Wochenschr. 49, 1271-1274.

6. Grasedyck, K., Ropohl, D., Szarvas, F. \& Lindner, J. (1971), Klin. Wochenschr. 49, 163-164.

7. Hanson, H. \& Mannsfeldt, H. G. (1971), Nova Acta Leopoldina Bd. 36 No. 200.

8. Kar, N. C. \& Pearson, C. M. (1972), Enzyme 13, 188-196.

9. Bowen, D. M. \& Davison, A. N. (1973), Biochem. J. 131, 417-419.

10. Swanson, A. A., Martin, B. J. \& Spicer, S. S. (1974), Biochem. J. 137, 223-228. daraus ergebenden Differenzierungsmöglichkeiten der Serum-Arylamidasen und -Peptidasen soll in einer weiteren Publikation eingegangen werden.

\section{Danksagung}

Wir danken Frau $H$. Frech für ihre fleißige und sorgfältige technische Assistenz.
11. Lorentz, K. Marunowski, A. \& Ritter, U. (1974), diese Z. $12,468-473$.

12. Lorentz, K., Petersen, S. \& Ritter, U. (1975), diese Z. 13, $45-48$.

13. Lorentz, K., Koch, C. D., Flatter, B. \& Molz, J. (1975), diese Z. 13, 49-52.

14. Bratton, A. C. \& Marshall, E. K. (1939), J. Biol. Chem. $128,537-550$

15. Richterich, R. (1971), Klinische Chemie, X. Auflage, S. 338-340, Verlag S. Karger, Basel.

16: Appel, W. (1970), „Aminopeptidasen und Aminosäurearylamidasen", in: Methoden der Enzymatischen Analyse (H. U. Bergmeyer ed.), X. Aufl., S. 916-921, Verlag Chemie, Weinheim.

17. Marshall, E. K. \& Litchfield, I. T. (1938), Science 88, 85-86.

18. Nagel, W., Willig, F. \& Schmidt, F. H. (1964), Klin. Wochenschr. 42, 447-449.
Prof. Dr. H. Struck Biochemische Abteilung Ostmerheimer Straße 200 D-5000 Köln 91 